\section{EFFECT OF NEUTRAL AND ACIDIC OLIGOSACCHARIDES ADMINISTRATION IN PRETERM INFANTS ON ALLERGIC DISEASES DURING THE FIRST YEAR OF LIFE}

\author{
N. Niele, E.A. Westerbeek, A. van Zwol, \\ H.N. Lafeber, R.M. van Elburg \\ Neonatology, VU University Medical Center, \\ Amsterdam, The Netherlands
}

Aim: Enteral administration of a prebiotic mixture of neutral and acidic oligosaccharides during the neonatal period in preterm infants may positively affect the Th2 response. Aim was to determine the effect of enteral supplementation of neutral and acidic (scGOS/IcFOS/AOS) oligosaccharides during neonatal period in preterm infants on the incidence of allergic diseases during the first year of life.

Methods: In a RCT, preterm infants $(\mathrm{GA}<32 \mathrm{wks}$ and/or BW< 1500g) were allocated to receive enteral scGOS/IcFOS/AOS supplementation or placebo (maltodextrin) between day 3 and 30 of life. Incidence of allergic disease (atopic dermatitis, bronchial hyperreactivity) was assessed by validated questionnaire. Data were analysed by logistic regression analysis.

Results: In total, 113 preterm infants were enrolled in the initial study. Of these 113, 99 were eligible for follow-up (11 died, 3 were excluded). To date, 75 infants participated in our follow-up study. Incidence of atopic dermatitis was not different in scGOS/ IcFOS/AOS- and placebogroup (5/37 (14\%)) versus $(4 / 38(11 \%))$ respectively $(p=0.69$, OR $1.3395 \% \mathrm{Cl}$ 0.33-5.39). Incidence of bronchial hyperreactivity was not different between scGOS/lcFOS/AOS- or placebogroup (10/37 (27\%) versus 12/38 (32\%)) respectively ( $p=0.67$, OR $0.8095 \% \mathrm{Cl} 0.30-2.18)$.

Adjustments for confounding factors (maternal education, parental history of atopy, smoking, and presence of pets at home) did not change the results of the primary analysis.

Conclusion: Enteral administration of a prebiotic mixture of acidic and neutral oligosaccharides during the neonatal period in preterm infants does not decrease the risk of atopic dermatitis or bronchial hyperreactivity during the first year of life.

\section{ENTERAL NUTRITION AND USE OF HUMAN MILK FORTIFIERS IN PRETERM INFANTS; AN INTERNATIONAL SURVEY}

C. Klingenberg ${ }^{1}$, N.D. Embleton ${ }^{2}$, S. Jacobs ${ }^{3}$, L. O'Connell ${ }^{3}, \mathrm{C}$. Kuschel ${ }^{3}$

${ }^{1}$ Dept. of Paediatrics, University Hospital of North Norway, Tromsø, Norway, ${ }^{2}$ Neonatal Services, Royal Victoria Infirmary, Newcastle Hospitals NHS

Foundation Trust, Newcastle upon Tyne, UK,

${ }^{3}$ Neonatal Services, Royal Women's Hospital, Melbourne, VIC, Australia

Background and aims: Optimal enteral nutrition is of critical importance to preterm infants, but there is limited evidence on benefits and safety of different feeding regimens including use of human milk fortifiers (HMF). We sought to determine feeding practice and use of HMF in neonatal units from different countries and continents.

Methods: An internet-based questionnaire was sent by email to a single neonatologist in 120 tertiary units in Australasia, Canada, Scandinavia and UK.

Results: 111 units (92\%) responded. Fifty-four units $(49 \%)$ had either a breast milk bank or access to donor milk (Australasia 2/24, Canada 6/25, Scandinavia 20/20 and UK 26/42). Enteral feeding was initiated in the first 24 hours of life in 42 units (38 \%) if GA $<25$ weeks, in 50 units ( $45 \%$ ) if GA 2527 weeks and in 81 units (73\%) if GA 28-31 weeks. For infants with $\mathrm{GA}<25$ weeks 55 units $(50 \%)$ used 4-5 days minimal enteral feeding before grading up feeding volume. Target feeding volume was $160-$ $180 \mathrm{ml} / \mathrm{kg} / \mathrm{d}$ in 62 units (56\%). Ninety units $(81 \%)$ routinely used a multi-component HMF. Most (85 $\%)$ used birth weight as criteria for which infants to be given HMF. Twenty-nine units added HMF when infants tolerated a feeding volume of $80-120 \mathrm{ml} / \mathrm{kg} /$ day and 43 units waited until infants tolerated "full feeds" ( $\geq 150 \mathrm{ml} / \mathrm{kg} /$ day).

Conclusions: This study examines key areas of uncertainty and shows that feeding practices demonstrate enormous variability. It provides data that will be useful in planning collaborative feeding trials. 